\title{
A modified coaxial electrospinning for preparing fibers from a high concentration polymer solution
}

\author{
D. G. Yu ${ }^{1 *}$, C. Branford-White ${ }^{2}$, K. White ${ }^{2}$, N. P. Chatterton ${ }^{2}$, L. M. Zhu ${ }^{1}$, L. Y. Huang ${ }^{1}$, B. Wang ${ }^{1}$ \\ ${ }^{1}$ College of Chemistry, Chemical Engineering and Biotechnology, Donghua University, Shanghai 201620, China \\ ${ }^{2}$ Institute for Health Research and Policy, London Metropolitan University, London, N7 8DB, UK
}

\begin{abstract}
A new process technology modified from conventional coaxial electrospinning process has been developed to prepare polymer fibers from a high concentration solution. This process involves a pure solvent concentrically surrounding polymer fluid in the spinneret. The concentric spinneret was constructed simply by inserting a metal needle through a high elastic silica gel tube. Two syringe pumps were used to drive the core polymer solution and the sheath solvent. Using polyvinylpyrrolidone (PVP) as the polymer model, which normally has an electrospinnable concentration of $10 \% \mathrm{w} / \mathrm{v}$ in ethanol, it was possible to electrospin $35 \% \mathrm{w} / \mathrm{v}$ of PVP in the same solvent, when pure N, N-dimethylacetamide (DMAc) was used as sheath fluid. The resultant fibers have a smooth surface morphology and good structural uniformity. The diameter of the fibers was $2.0 \pm 0.25 \mu \mathrm{m}$ when the DMAc-to-polymer-solution flow rate ratio was set as 0.1 . The process technology reported here opens a new window to tune the polymer fibers obtained by the electrospinning, and is useful for improving productivity of the electrospinning process.
\end{abstract}

Keywords: processing technologies, coaxial electrospinning, polymer fibers, productivity

\section{Introduction}

Polymer fibers provide a platform for a number of diverse applications, including drug encapsulation, biosensing, filtration, fuel cells, composites, and nanoelectronics [1-3]. Among the advanced processing techniques that have been used to produce fibers from natural and synthetic polymers, the electrospinning process provides the simplest approach to prepare nano- or microscale diameter fibers that are exceptionally long, uniform in shape, and diverse in composition [4-6]. By utilizing electrostatic interactions and elongation of the viscoelastic jet system, electrospinning can prepare ultrafine fibers from a polymer solution or molten sample adopting a simple one-step process. Numerous polymers have been reported to be electrospun into nanofibers for functional applications in the literature [1-6].
However, although the one-step 'top-down' electrospinning process is simple, there is a wide range of parameters that influence the electrospinnability of polymer solutions and properties of the generated fibers. These parameters include solution properties (concentration, viscosity, elasticity, conductivity, and surface tension); systematic variables (flow rate, electric potential at the capillary tip, and the distance between the tip and the fiber collector); and ambient conditions (temperature, humidity, and air velocity) $[7,8]$. Among these parameters, concentration is regarded as the most significant parameter for developing smooth electrospinning and high quality fibers $[9,10]$.

Fiber formation is primarily governed by the viscosity of the polymer solution and strength of the applied electric field. The viscosity of the polymer solution depends on the concentration and structure

\footnotetext{
${ }^{*}$ Corresponding author, e-mail: ydg017@dhu.edu.cn
}

(c) BME-PT 
of the polymer. Often there is an upper and lower limit for polymer concentration during electrospinning [11]. It is well known that the prerequisite for fiber formation is the presence of sufficient chainentanglement density in the working solution as this prevents the capillary breakup and provides deformable networks. When the polymer concentration is lower than the minimum, there is insufficient chain-entanglement for filament forming and electrospinning will degenerate into electrospraying. A number of studies have demonstrated this phenomenon $[12,13]$. However, little research has been undertaken to determine the upper concentration limit for smooth electrospinning. Often it is not possible to electrospin at high polymer concentrations due to viscosity factors that influence solvent levels and so affect the formation of a stable jet, thereby clogging the flow of components required for continuous electrospinning. If polymer solution with a high concentration can be electrospun into ultra-fine fibers, it would inevitably improve the capability of electrospinning and broaden the applications of polymer fibers. Examples include: extending the range of electrospinnable concentrations of polymer solutions; improving the productivity of the electrospinning process; allowing preparation of ultra-fine fibers with diameters between several microns to $50 \mu \mathrm{m}$ for biomedical applications such as improving cell penetration effects of fiber-based scaffolds; facilitating encapsulation of functional materials in a core-sheath manner.

On the other hand, although significant progress has been made in the electrospinning process over the past few years and the resultant nanostructures have been exploited in a wide range of applications, this simple one-step top-down nanofiber fabricating process still needs to be optimized so as to broaden its filament-forming capability over a wide range of materials and with higher nanofiber production rates $[14,15]$.

Recently there is a growing need for fiber materials that are flexible and provide opportunities for the development of new electrospinning techniques that include specialized nozzle systems, auxiliary apparatus, guiding electrodes and functionalized target electrodes [16, 17]. These electrospinning processes include coaxial electrospinning, side-byside electrospinning, elevated temperature electrospinning, vibrational electrospinning, magneto- electrospinning, siroelectrospinning and bubble electrospinning, and electrospinning with an airblowing system, electrospinning in raised humidity conditions, and electrospinning in vacuum conditions for increasing the controllablity of the filament-forming process and also for improving the quality of the resultant fibers [18-24]. Nonetheless, seldom effort has been spent on overcoming and understanding the limitations of high polymer concentration for smooth electrospinning. Although there are several recent studies aimed at preventing clogging during the conventional electrospinning process $[25,26]$, they did not investigate the possibility of electrospinning polymer solutions with high concentrations that normally is impossible for the conventional electrospinning process.

In the present study, a new modified coaxial electrospinning process was developed to extend the upper concentration limit of electrospinnable solutions of polyvinylpyrrolidone (PVP), a hydrophilic filament-forming polymer used in our laboratory previously [27-29], that has a wide variety of applications in medicine, food, pharmacy, cosmetics and industrial production [30]. The new process involves synchronously pumping pure solvent concentrically around the high concentration polymer solutions to facilitate the electrospinning process.

\section{Materials and methods}

\subsection{Materials}

PVP K60 $\left(M_{\mathrm{w}}=360000\right)$ was purchased from Shanghai Yunhong Pharmaceutical Aids and Technology Co., Ltd. (Shanghai, China). Analytic grade $\mathrm{N}, \mathrm{N}$-dimethylacetamide (DMAc) and anhydrous ethanol was provided by the Sinopharm Chemical Reagent Co., Ltd. Water was highly purified water.

\subsection{Electrospinning process}

Spinning solutions of PVP of concentrations 10, 30 and $35 \% \mathrm{w} / \mathrm{v}$ were prepared in anhydrous ethanol, respectively. The solutions at concentration of 10 and $30 \%$ were used in conventional electrospinning.

A schematic diagram and photographs of the modified coaxial electrospinning apparatus are shown in Figure 1. Two syringe pumps (KDS100 and KDS200, Cole-Parmer, Illinois, USA) were used for driving the high concentration PVP solutions and the surrounding DMAc solvent synchronously. 


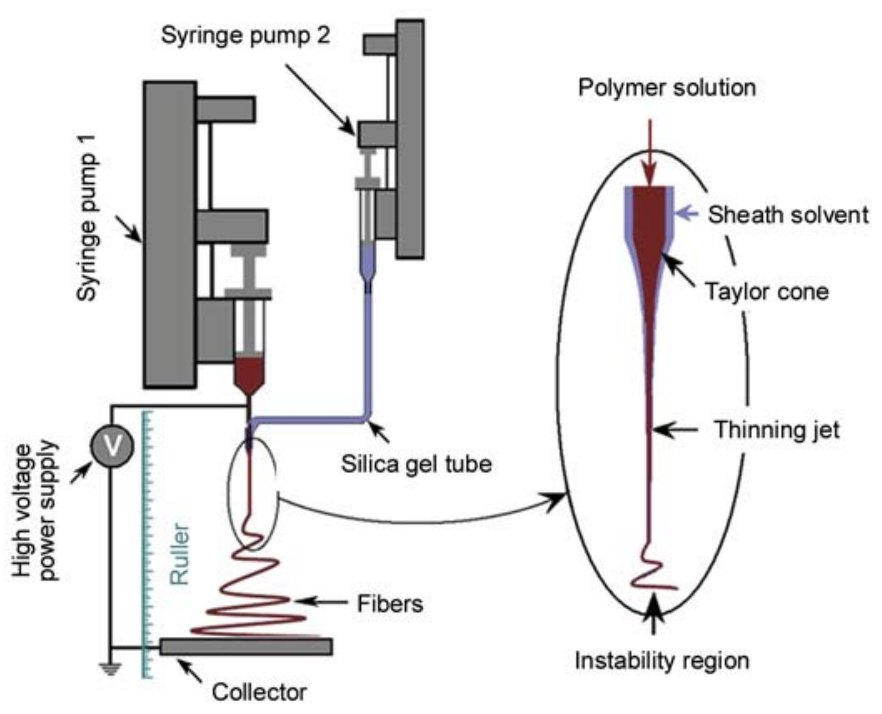

a)
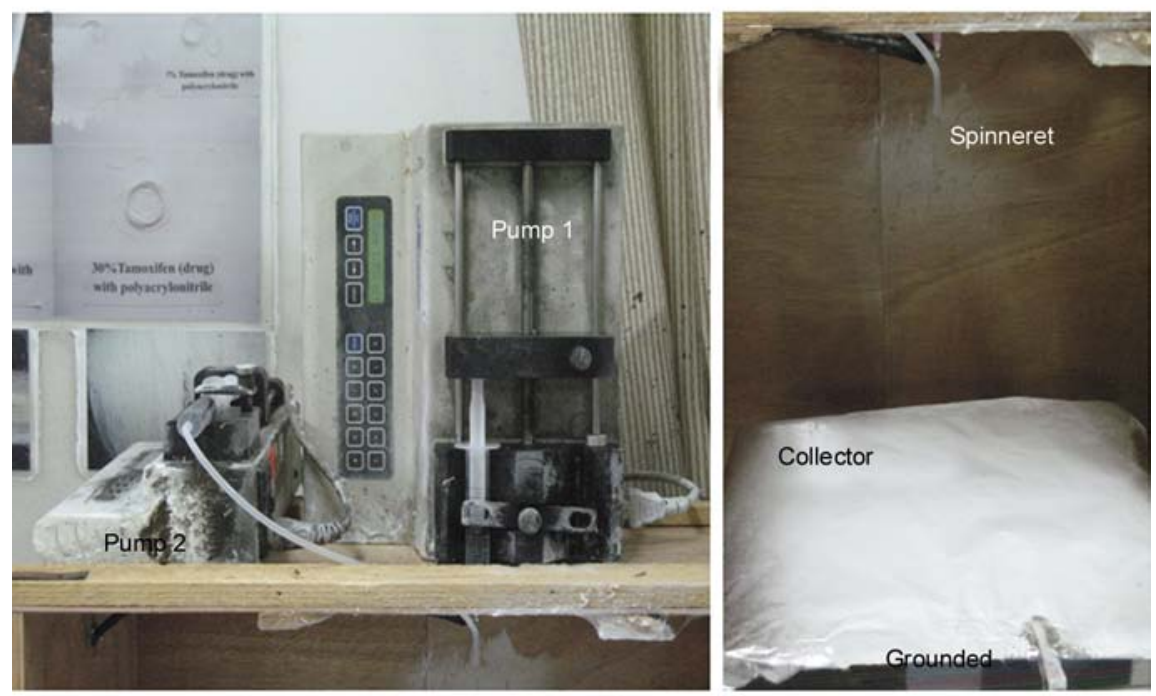

b)

c)

Figure 1. An apparatus for modified coaxial electrospinning. a) schematic diagram, b) pump, c) spinneret and collector.

A high-voltage power supply (ZGF $60 \mathrm{kV} / 2 \mathrm{~mA}$, Shanghai Sute Corp., China) was used at a fixed voltage of $10 \mathrm{kV}$, and the fibers produced were collected on aluminium foil at a pre-determined distance. The core and sheath flow rate were selected as 1.0 and $0.1 \mathrm{ml} / \mathrm{h}$ respectively. All electrospinning was carried out under ambient conditions $\left(24 \pm 2^{\circ} \mathrm{C}\right.$ with relative humidity $54 \pm 4 \%$ ).

Electrospinning was recorded using a digital video recorder (maximum magnification $16 \times$, PowerShot A640, Canon, Tokyo, Japan). The fibers were dried for over $24 \mathrm{~h}$ at $40^{\circ} \mathrm{C}$ under vacuum $(320 \mathrm{~Pa}$ ) in a DZF-6050 Electric Vacuum Drying Oven (Shanghai Laboratory Instrument Work Co. Ltd., Shanghai, China) to facilitate the removal of residual organic solvent and moisture.

\subsection{Characterization}

Surface morphology of the electrospun fibers was observed using an XP-700 polarized optical microscope (PM, Shanghai Changfang Optical Instrument Co., Ltd) or a JSM-5600LV scanning electron microscope (SEM, Japan Electron Optics Laboratory Co. Ltd., Tokyo, Japan). Prior to SEM examination, samples were gold sputter-coated under argon to render them electrically conductive. Pictures were then taken at an excitation voltage of $10 \mathrm{kV}$. The average fiber diameter was determined by measuring diameters of fibers at over 100 points from SEM images or polarized microscopic images using Image J software (National Institutes of Health, USA). 


\section{Results and discussion}

\subsection{Conventional electrospinning of $\mathbf{1 0}$ and 30\% PVP solutions}

When PVP solutions with a concentration of $30 \%$ flow from the syringe to the needle tip in the absence of an electric field, a semispherical droplet

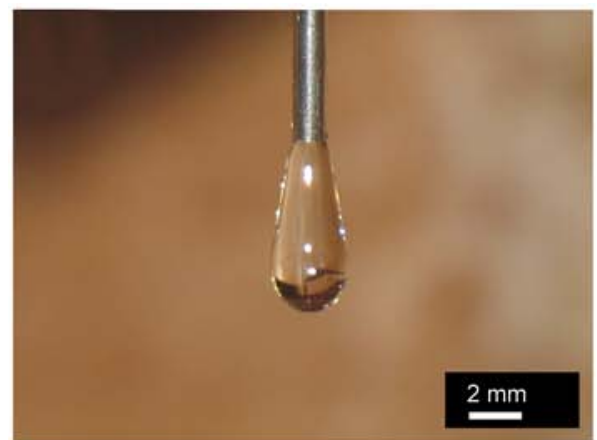

a)

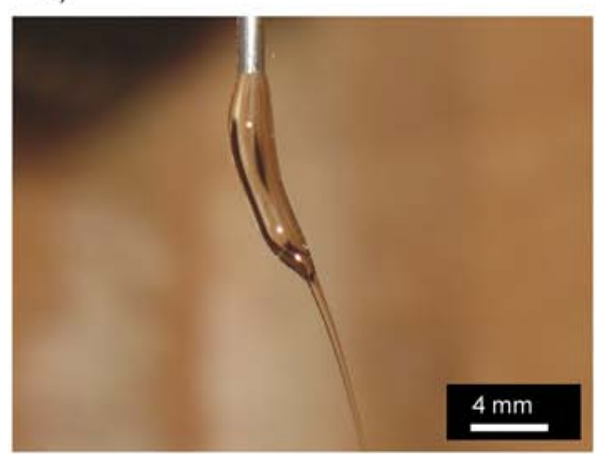

c)

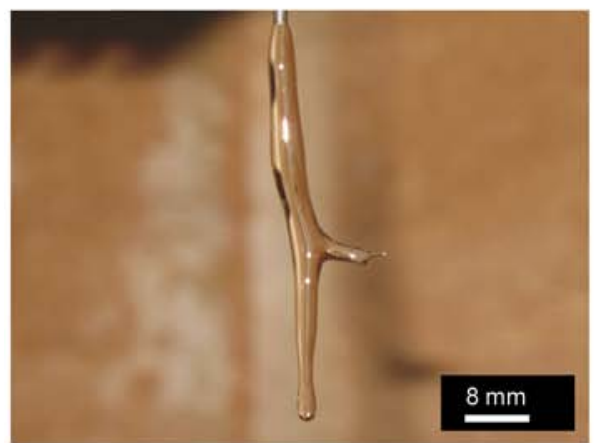

e)

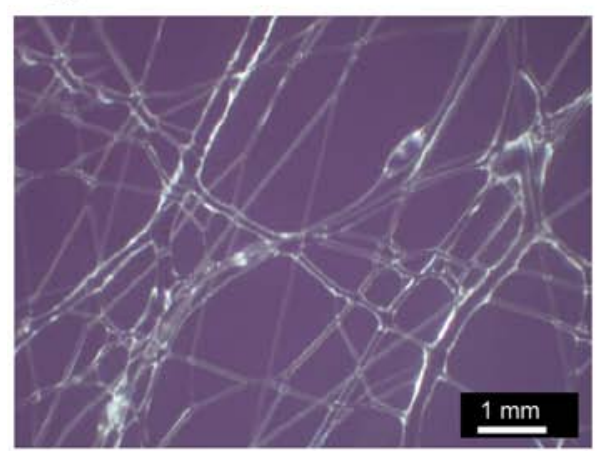

g) forms at the needle tip due to the effect of the surface tension of the solution (Figure 2a). The droplet size becomes larger and longer with a continuous supply of the polymer solution, and finally forms a column, which hangs on the tip until the gravitational force overcomes the surface tension, at which

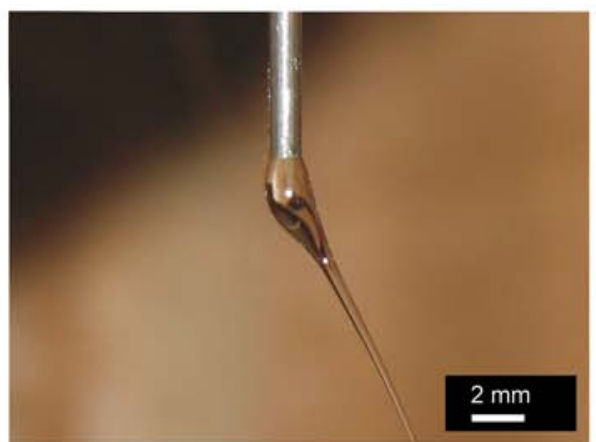

b)

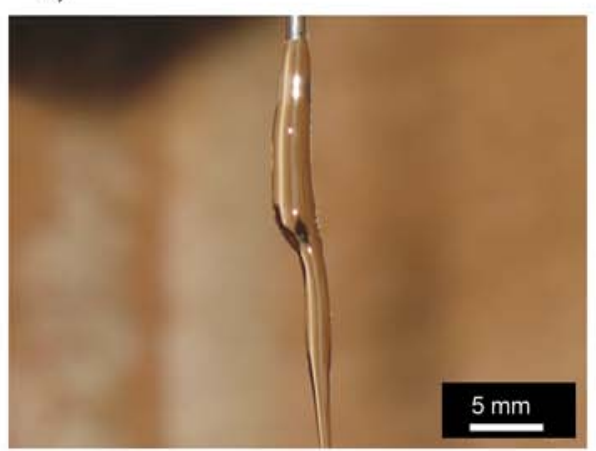

d)

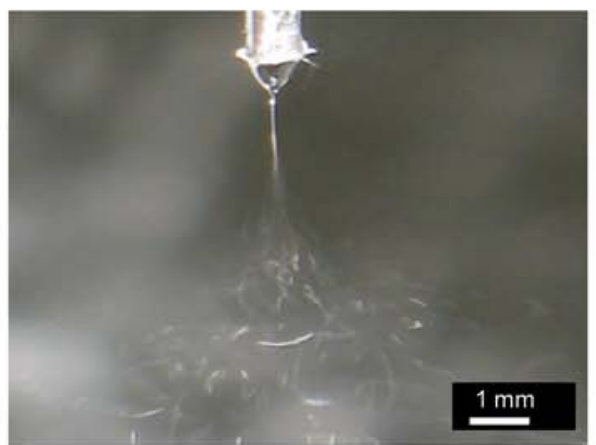

f)

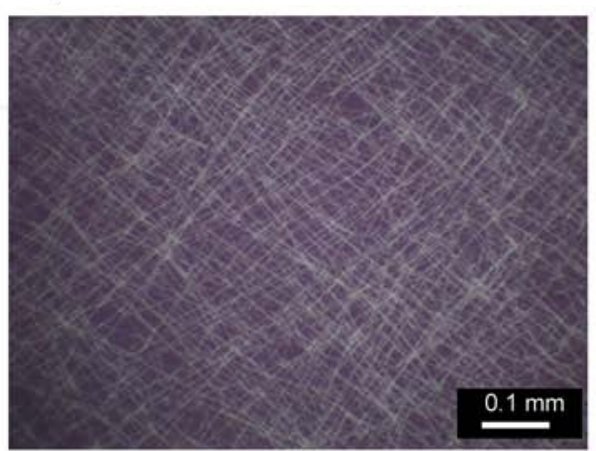

h)

Figure 2. Images of conventional electrospinning process and the resultant fibers. a) to e) $30 \%(w / v)$ PVP solutions being electrospun in an open atmosphere, f) $10 \%(\mathrm{w} / \mathrm{v})$ PVP solution being electrospun in an open atmosphere, $\mathrm{g}$ ) and h) polarized microscopic images of the resultant fibers from 30 and 10\% PVP solutions, respectively. 
point the column then drips from the needle tip and new droplet and column form. During the formation and growth of the column, solvent evaporation naturally occurs due to the difference between the concentration of the solvent in the solution and that in the atmosphere. When the polymer solution was subjected to an electrically charge of $10 \mathrm{kV}$, the polymer droplet shape began to change from a semi-spherical to a conical shape, and a jet was formed because the electric force overcame the shape-maintaining forces from surface tension (Figure $2 b$ ).

As time continued, a column formed and grew rapidly (Figure $2 \mathrm{c}$ and $\mathrm{d}$ ). The high viscosity of PVP solutions at high concentrations and the highvolatility of the solvent ethanol promoted the quick formation of an even more viscous gel-like skin on the droplet surface. The applied electrical force was not adequate to overcome the viscous drag force at the droplet-air interface; meanwhile the core was still fluid and driven by solution input from the pump, which in turn led to the formation of a column and a gradual enlarging of the gel skin and the volume of column. The fast evaporation of ethanol was due to Joule heating (resulting from volumetric heating when an electric field is applied across a conducting medium such as a polymer solution [31]) and the difference in concentration of ethanol between the polymer solution and the open atmosphere.

As the column further grew, the Taylor cone moved forward along the column ending up far from the tip of spinneret. This gave the solvent a long time to evaporate. Thus it was inevitable that the polymer solution could no longer be ejected from the Taylor cone, and hence the electrospinning jet was interrupted (Figure 2e). After gravitational and electronic forces cleaned up the column, elctrospinning could continue but only for a short time, after which it stopped again. Electrospinning of the high concentration PVP solution could not be carried out smoothly and continuously.

Shown in Figure $2 \mathrm{~g}$ are electrospun fibers collected at a distance of $25 \mathrm{~cm}$ from the top of spinning head. The fibers obviously had very poor quality (a wide ranging diameter distribution, and all fibers bonded together) resulting from the instability of electrospinning of high PVP concentration solutions. Such clogging is frequently observed when high-volatility solvents are used to prepare polymer solutions [32, 33]. Clogging of 30\% PVP solutions was accelerated owing to the high viscosity of the solution, and in contrast 10\% PVP solutions could be electrospun smoothly and continuously (Figure 2f), and the resultant nanofibers were homogeneous (Figure 2h).

\subsection{Coaxial electrospinning with sheath solvent}

To attempt electrospinning of concentrated solutions of PVP, we used a coaxial arrangement in which the PVP solution was spun through a stainless steel capillary metal-hub needle (with an inner and outer diameter of 1 and $1.32 \mathrm{~mm}$ respectively) inserted into a silica gel tube (with an outer and inner diameter of 4 and $2 \mathrm{~mm}$ respectively) (Figure 3a).

Sheath solvent, DMAc, was coaxially spun around the PVP solution. Using this arrangement it was possible to electrospin fibers from a concentrated PVP solution of $35 \%(\mathrm{w} / \mathrm{v})$, which would not be possible when using a conventional electrospinning apparatus. In the absence of sheath solvent a droplet formed immediately when the PVP solution was squeezed out of the needle (Figure 3b), which was due to the evaporation of ethanol in the atmosphere, and high viscoelasticity and surface tensions of the solution.

When sheath solvent was supplied to surround the high concentration PVP solution, a half ellipse droplet was formed (Figure 3c). When a voltage of $10 \mathrm{kV}$ was supplied, the droplet shape changed from semi-spherical to conical and a jet was formed stably under the electrical force (Figure 3d).

By using a DMAc flow rate that was only $10 \%(\mathrm{w} / \mathrm{v})$ of the PVP solution, the electrospinning of the high concentration PVP solution was continuous and smooth. The surrounding DMAc played the following roles during the electrospinning process: (1) lubricating the spinning head to avoid the polymer clinging to it; (2) slowing the rate of solvent evaporation and hence retarding the formation of a highly viscous surface on the Taylor cone and the thinning jet; (3) keeping the jet at a fluid state and thus under an electrical drawing force for a longer time to make the fiber diameters thinner.

It is well known that electrospinning involves the use of an electrical charge to draw micro or nano 


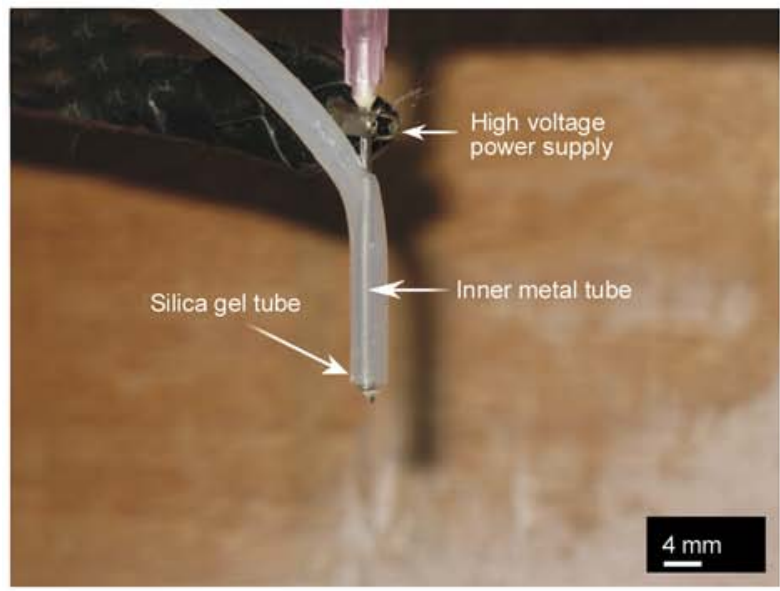

a)

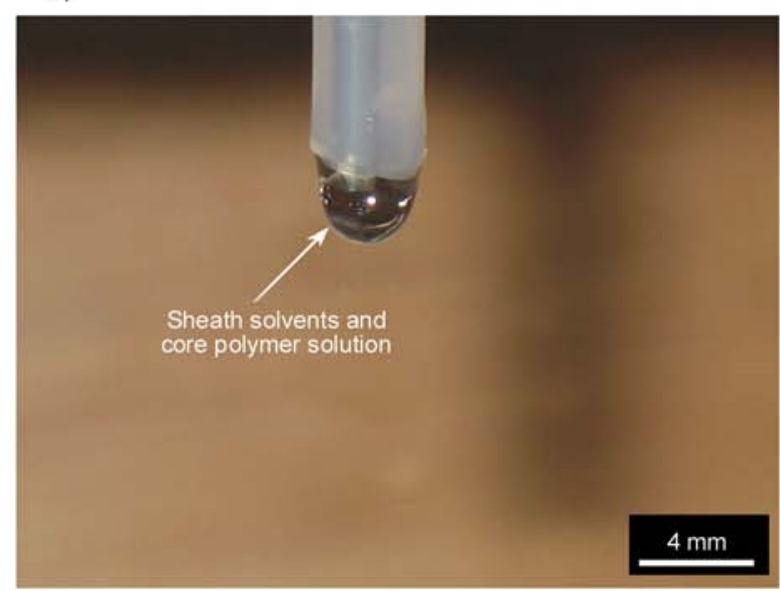

c)

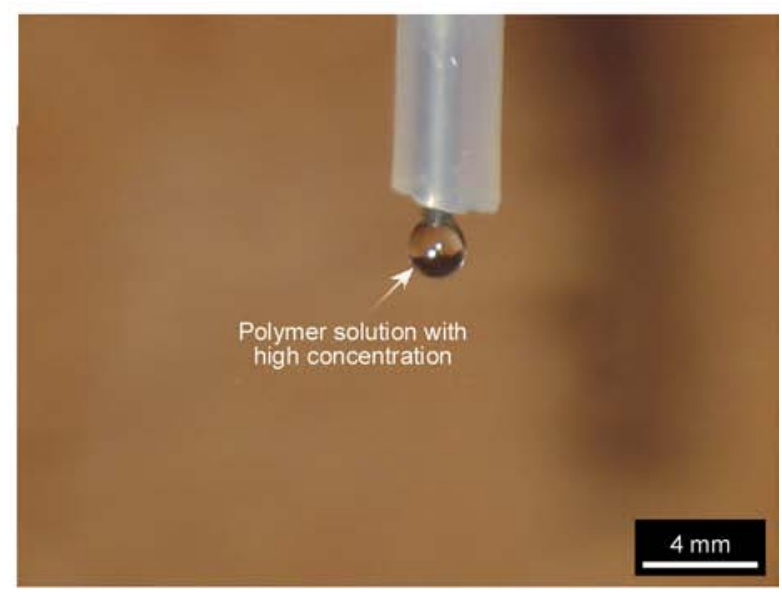

b)

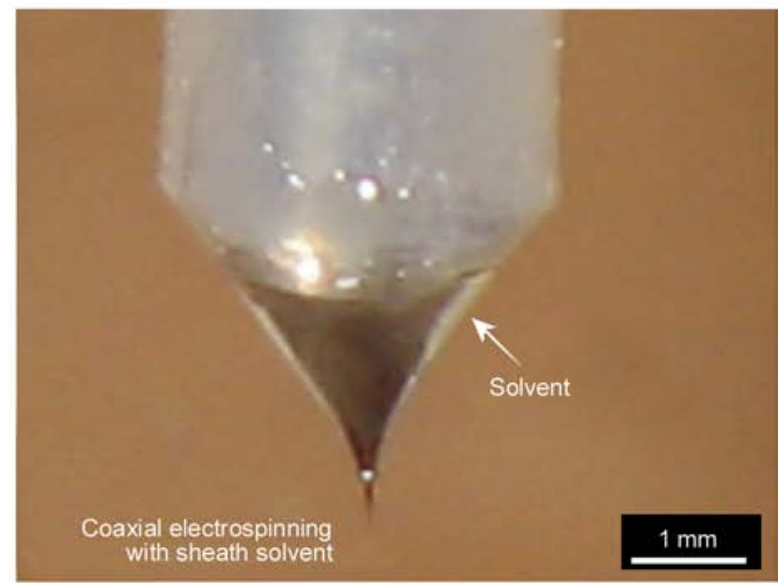

d)

Figure 3. Modified coaxial electrospinning. a) Arrangement of spinneret, b) and c) $35 \%$ (w/v) PVP droplets without and with sheath DMAc, respectively, d) morphologies of a Taylor cone and jet formed in the electrical field using a DMAc-to-PVP-solution flow rate ratio of 0.1 .

scale fibers from a liquid and the process shares characteristics of both electrospraying and conventional solution dry spinning of fibers. In the present electrospinning process, the formation of the Taylor cone, and the thinning of the jet occurred when they were surrounded by a solvent, instead of the ambient atmosphere in the conventional electrospinning process. The formation of gel-like skin and clogging of the spinning head that results from conventional electrospinning of high concentration polymer solutions could be retarded by preventing the loss of polymer solution solvent at the droplet-air and jet-air interface effectively, using an additional solvent as sheath fluid.

\subsection{Fibers collected at different positions from the spinneret}

Figure $4 \mathrm{a}$ to e shows optical images of electrospun fibers that were prepared from 35\% (w/v) PVP solution using the modified coaxial electrospinning process and collected at a distance of 5, 15, 18, 22 and $25 \mathrm{~cm}$ from the top of spinning head, respectively. A rule (Figure 1) was used to indicate the sampling place during the electrospinning process.

The fibers shown in Figure 4a were collected from the straight thinning jet $(5 \mathrm{~cm})$, and had an estimated diameter of about $93 \mu \mathrm{m}$. The fibers shown in Figure $4 \mathrm{~b}$ were collected just before the onset point of the instability regions $(15 \mathrm{~cm})$; their diameters were about $72 \mu \mathrm{m}$. Although there was a relatively long distance $(10 \mathrm{~cm})$ between the two collection points, a thinning effect on the fibers was not obvious. The fibers shown in Figure 4c were collected at on the onset point of whipping and bending $(18 \mathrm{~cm})$; they had an estimated diameter of $29 \mu \mathrm{m}$. At this point, the jet was subject to a number of electrically driven bending instabilities that caused the fluid path to become a series of coiled 
coils [34], resulting in the special fiber morphology observed here.

All the fibers shown in Figure 4a, b and c were still 'wet'. They had a flat morphology and were bonded together at joint points, suggesting that the true fiber diameter is a little smaller than the measured

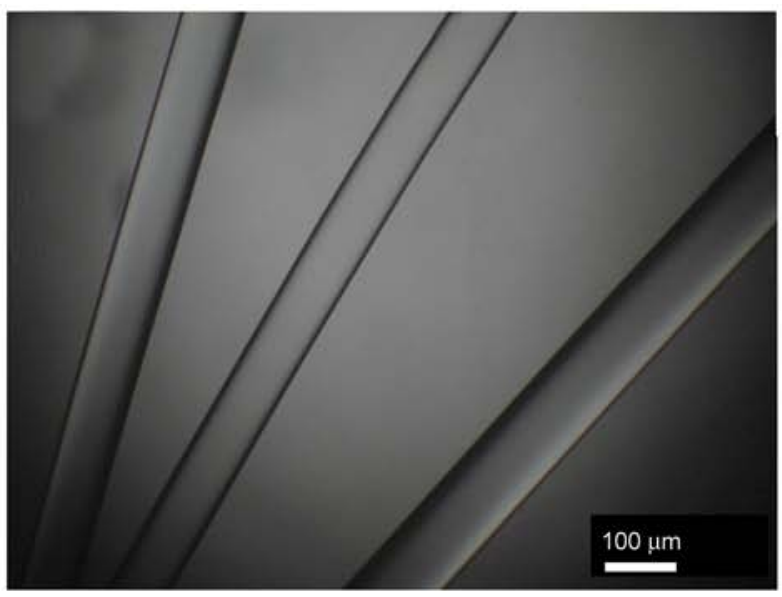

a)

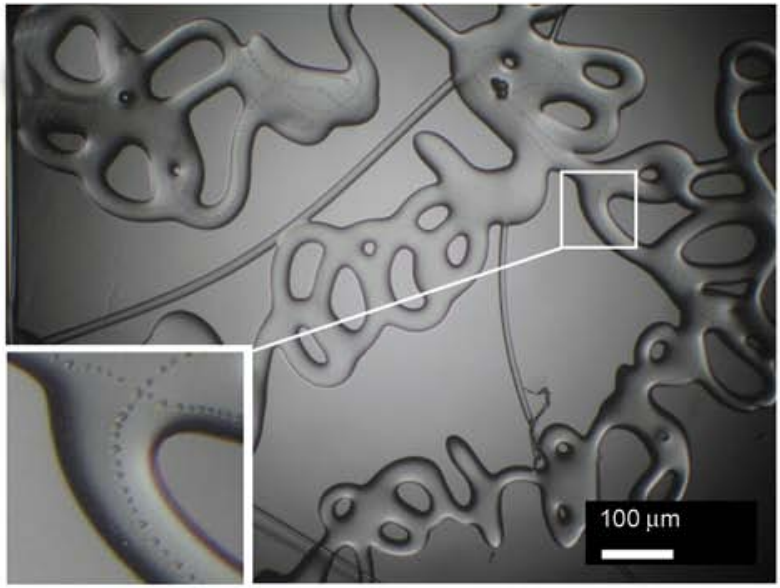

c)

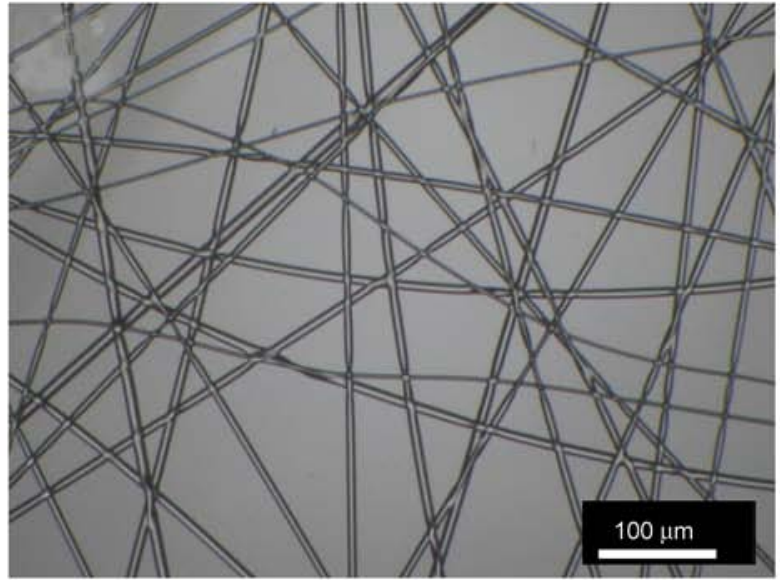

e) data. In addition, unusual bubbles were observed on the fibers shown in Figure $4 \mathrm{~b}$ and $\mathrm{c}$ (inset). This phenomenon can be attributed to evaporation of the inner solvent during the thinning jet flight and drawing processes. The presence of these bubbles indicate that the surface of the jet fluids was differ-

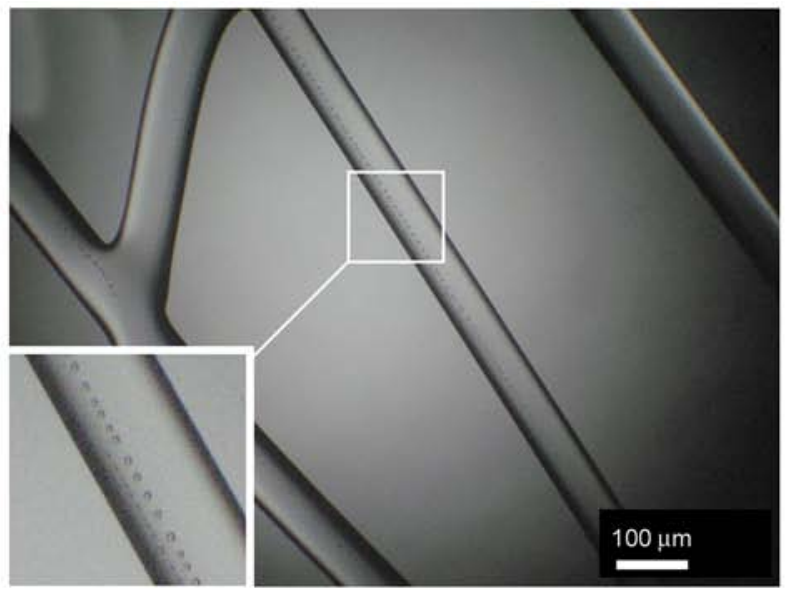

b)

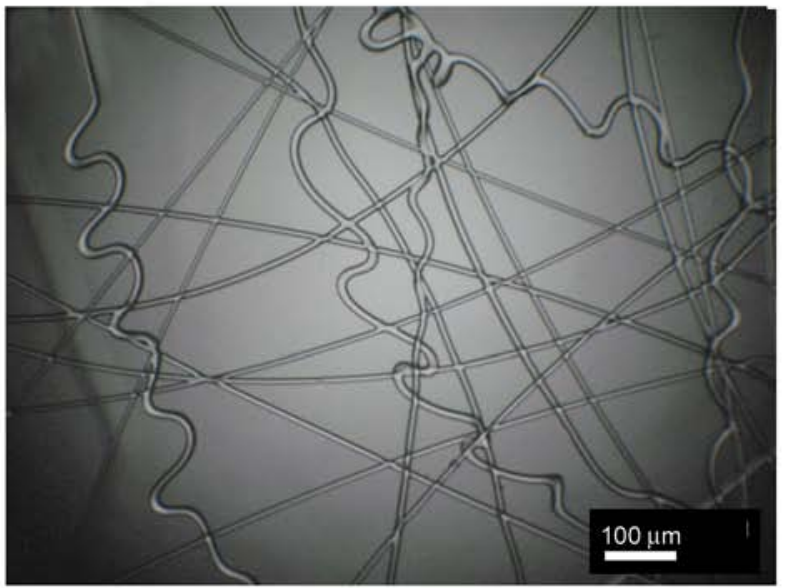

d)

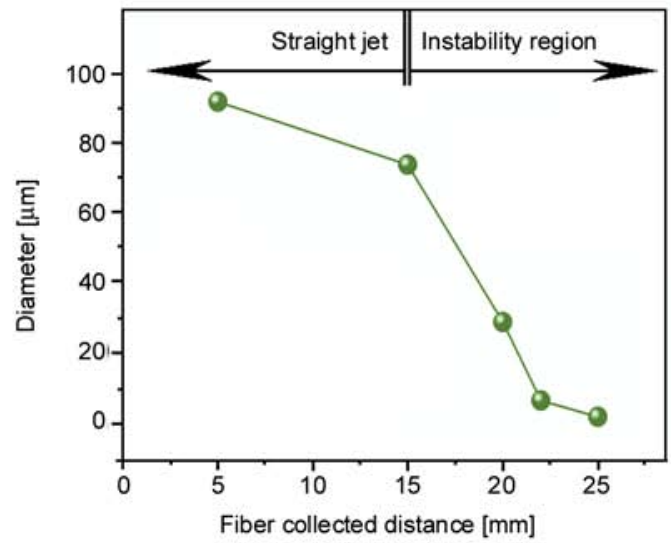

f)

Figure 4. Polarized microscopic images of fibers collected at different positions from the spinning head (a) to e)), and the observed change in fiber diameter with collection distance. a) to e) correspond to a distance of $5,15,18,22$ and $25 \mathrm{~cm}$, respectively. 
ent from their core, and was still soft enough for the inner solvent to penetrate out and evaporate during the drawing process.

Figure $4 \mathrm{f}$ shows how collection distance affects fiber diameter. In the thinning jet region of the plot the fibers' diameter decreased to only a small extent mainly due to the drawing effect of the vertical electric force and to evaporation of the solvents. At longer collection distances, the fiber diameters decreased dramatically to several micrometers after the onset of the instability region. It is the electronic repulsion from Coulombic force occurred on the polymer jet surface that mainly expanded the fiber circular cross-sections and created the significant elongation of straight fibers before homogeneous fiber solidification. The whipping and bending processes were similar to those in the traditional electrospinning process. The surrounding DMAc had kept them at a fluid state for elongation in the

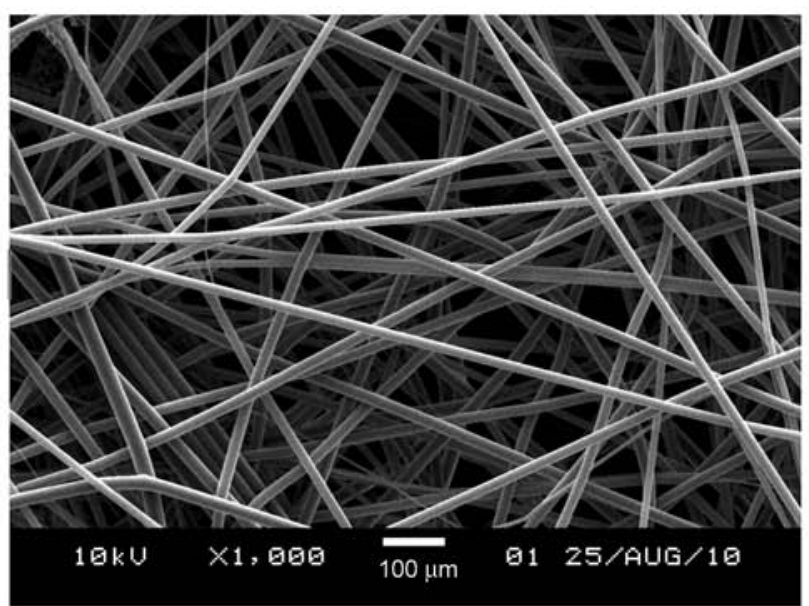

a)

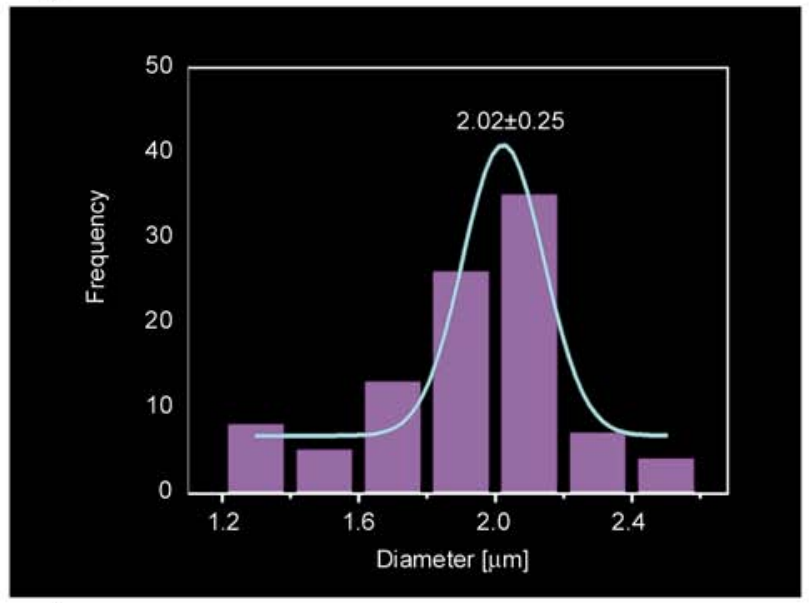

c) instability region. At even longer collection distances, the decrease of fiber diameters would slow down or till stop mainly due to decrease of charge densities, evaporation of most of the solvent and gradual solidification of the fibers [34].

Figure 5a and $\mathrm{b}$ show SEM images of fibers collected at a distance of $28 \mathrm{~cm}$ from the nozzle of the spinneret. The fibers have a smooth surface, and homogeneous structure with an average diameter of $2.02 \pm 0.25 \mu \mathrm{m}$ (Figure 5c). Figure 5d shows SEM images of fibers from $10 \%$ PVP solutions, which has an even wider fiber diameter distribution $(140 / 650 \cdot 100 \%=22 \%)$ than fibers from the modified coaxial electrospinning process $(250 / 2020 \cdot 100 \%=12 \%)$. Coaxial electrospinning with pure solvent as sheath fluid makes the process, the formation of Taylor cone and the thinning straight jet more stable than a single fluid electrospinning process conducted in an open atmosphere.

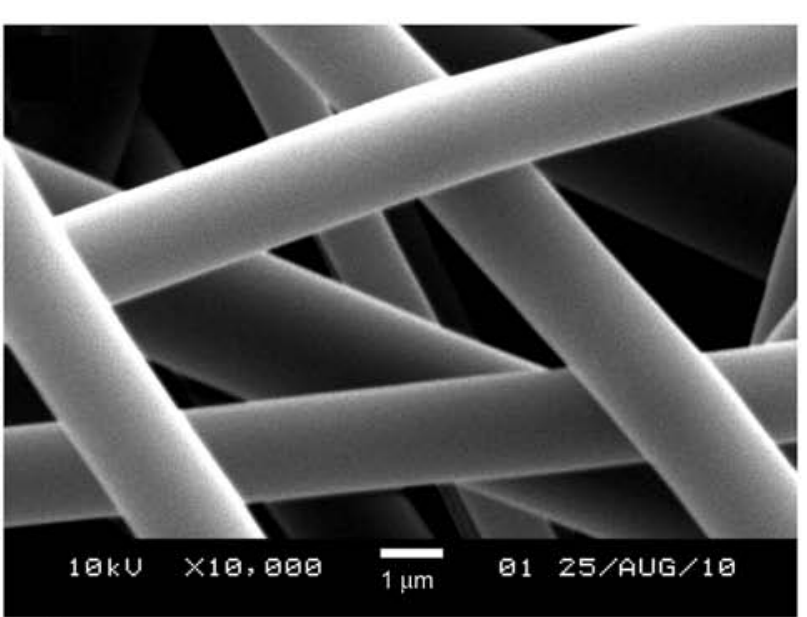

b)

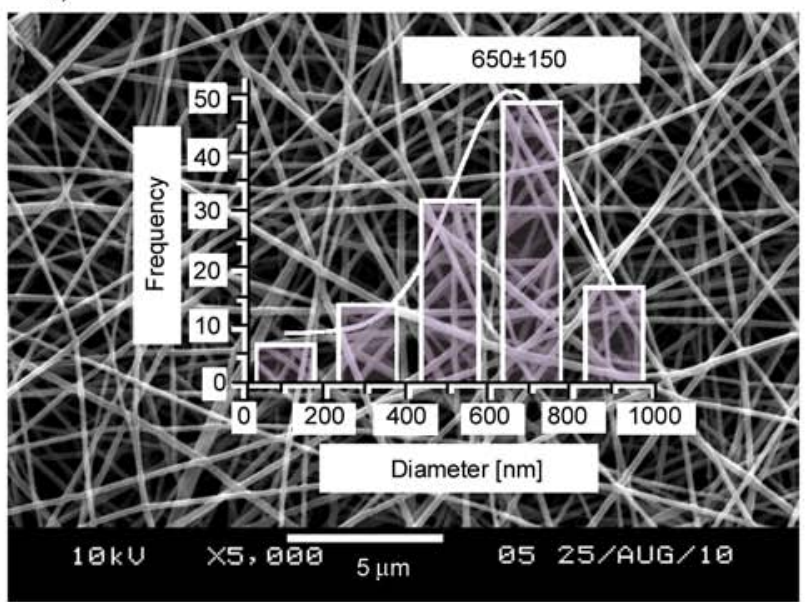

d)

Figure 5. SEM images of the ultra-fine fibers. a) and b) fibers prepared by the modified coaxial electrospinning from $35 \%(w / v)$ PVP solutions with DMAc as sheath fluid and a DMAc-to-PVP-solution flow rate ratio of 0.1, under different magnifications, c) fiber diameter distribution, d) fibers prepared from 10\% w/v PVP solutions using the traditional single fluid electrospinning. 


\section{Conclusions and outlooks}

This study investigated a new process technology modified from coaxial electrospinning for preparing ultrafine fibers from high concentration polymer solutions. The modified process is characterized by a solvent surrounding a polymer solution in the spinneret, and can be easily assembled by simply using a metal needle to penetrate through an elastic silica gel tube and using two syringe pumps. PVP, which has a normal electrospinnable concentration of $10 \%$ in ethanol, was successfully spun into ultrafine fibers from a solution with a concentration of $35 \%(\mathrm{w} / \mathrm{v})$ using the process developed here with DMAc as the surrounding solvent. Polarized microscopic observations revealed the process of formation of ultrafine fibers. They verified that the surrounding DMAc effectively facilitated the electrospinning process through retarding the fast evaporation of ethanol from the Taylor cone and the straight thinning jet. The SEM results demonstrated that the resultant fibers had smooth surface morphology and high structural uniformity.

The modified coaxial electrospinning process reported here can open a new window to tune the polymer fibers obtained by the electrospinning and improve its capability in processing polymer materials. It is obvious that electrospinning with a surrounding solvent will bring out more and new possibilities than traditional electrospinning process in air. For example, it may: (1) facilitate the electrospinning process through eliminating the clogging of spinneret that is often taken place in the electrospinning of spinning solutions in a low boiling point organic solvent; (2) extend the range of electrospinnable concentrations of polymer solutions; (3) improve the productivity of the electrospinning process; (4) enable to prepare nanofibers from polymers without electrospinnability owing to lack of suitable solvents through coagulating in a surrounding solvent environment; (5) enable to prepare nanofibers from polymers without electrospinnability owing to dilute concentration through extraction of the abundant solvent in the core solution using a poor solvent of the polymer as sheath fluid.

\section{Acknowledgements}

We would like to thank the China Postdoctoral Science Foundation (NO. 200902195) and UK-CHINA Joint Laboratory for Therapeutic Textiles for financial supports.

\section{References}

[1] Greiner A., Wendorff J. H.: Electrospinning: A fascinating method for the preparation of ultrathin fibers. Angewandte Chemie International Edition, 46, 56705703 (2007).

DOI: $10.1002 /$ anie. 200604646

[2] Nagy Zs. K., Nyúl K., Wagner I., Molnár K., Marosi Gy.: Electrospun water soluble polymer mat for ultrafast release of Donepezil HCl. Express Polymer Letters, 4, 763-772 (2010).

DOI: $10.3144 /$ expresspolymlett.2010.92

[3] Yu D-G., Shen X-X., Branford-White C., White K., Zhu L-M., Bligh S. W. A.: Oral fast-dissolving drug delivery membranes prepared from electrospun PVP ultrafine fibers. Nanotechnology, 20, 055104/1055104/9 (2009).

DOI: $10.1088 / 0957-4484 / 20 / 5 / 055104$

[4] Yu D-G., Gao L-D., White K., Branford-White C., Lu W-Y., Zhu L-M.: : Multicomponent amorphous nanofibers electrospun from hot aqueous solutions of a poorly soluble drug. Pharmaceutical Research, 27, 2466-2477 (2010).

DOI: $10.1007 / \mathrm{s} 11095-010-0239-\mathrm{y}$

[5] Rutledge G. C., Fridrikh S. V.: Formation of fibers by electrospinning. Advanced Drug Delivery Review, 59, 1384-1391 (2007).

DOI: $10.1016 /$ j.addr.2007.04.020

[6] Dhanalakshmi M., Jog J. P.: Preparation and characterization of electrospun fibers of Nylon 11. Express Polymer Letters, 2, 540-545 (2008).

DOI: 10.3144 /expresspolymlett.2008.65

[7] Heikkilä P., Harlin A.: Electrospinning of polyacrylonitrile (PAN) solution: Effect of conductive additive and filler on the process. Express Polymer Letters, 3, 437-445 (2009).

DOI: $10.3144 /$ expresspolymlett.2009.53

[8] Tripatanasuwan S., Zhong Z., Reneker D. H.: Effect of evaporation and solidification of the charged jet in electrospinning of poly(ethylene oxide) aqueous solution. Polymer, 48, 5742-5746 (2007). DOI: $10.1016 /$ j.polymer.2007.07.045

[9] Gupta P., Elkins C., Long T. E., Wilkes G. L.: Electrospinning of linear homopolymers of poly(methyl methacrylate): Exploring relationships between fiber formation, viscosity, molecular weight and concentration in a good solvent. Polymer, 46, 4799-4781 (2005). DOI: $10.1016 /$ j.polymer.2005.04.021

[10] Cengiz F., Dao T. A., Jirsak O.: Influence of solution properties on the roller electrospinning of poly(vinyl alcohol). Polymer Engineering and Science, 50, 936943 (2009).

DOI: $10.1002 /$ pen.21599

[11] Liu Y., He J-H., Yu J-Y., Zeng H-M.: Controlling numbers and sizes of beads in electrospun nanofibers. Polymer International, 57, 632-636 (2008).

DOI: $10.1002 /$ pi.2387 
[12] Wang C., Chien H-S., Hsu C-H., Wang Y-C., Wang CT., Lu H-A.: Electrospinning of polyacrylonitrile solutions at elevated temperatures. Macromolecules, 40, 7973-7983 (2007).

DOI: $10.1021 / \mathrm{ma} 070508 \mathrm{n}$

[13] Reneker D. H., Yarin A. L., Fong H., Koombhongse S.: Bending instability of electrically charged liquid jets of polymer solutions in electrospinning. Journal of Applied Physics, 87, 4531-4547 (2000).

DOI: $10.1063 / 1.373532$

[14] Varesano A., Carletto R. A., Mazzuchetti G.: Experimental investigations on the multi-jet electrospinning process. Journal of Materials Processing Technology, 209, 5178-5185 (2009).

DOI: $10.1016 /$ j.jmatprotec.2009.03.003

[15] Demir M. M.: Investigation on glassy skin formation of porous polystyrene fibers electrospun from DMF. Express Polymer Letters, 4, 2-8 (2010). DOI: $10.3144 /$ expresspolymlett.2010.2

[16] Park S., Park K., Yoon H., Son J. G., Kim G. H.: Apparatus for preparing electrospun nanofibers: Designing an electrospinning process for nanofiber fabrication. Polymer International, 56, 1361-1366 (2007). DOI: $10.1002 /$ pi.2345

[17] Wang M., Yu J-H., Kaplan D. L., Rutledge G. C.: Mechanical properties of electrospun silk fibers. Macromolecules, 37, 6856-6864 (2004).

DOI: $10.1021 / \mathrm{ma} 048988 \mathrm{v}$

[18] Zhang J-F., Yang D-Z., Xu F., Zhang Z-P., Yin R-X., Nie J.: Electrospun core-shell structure nanofibers from homogeneous solution of poly(ethylene oxide)/ chitosan. Macromolecules, 42, 5278-5284 (2009). DOI: $10.1021 / \mathrm{ma} 900657 \mathrm{y}$

[19] Gupta P., Wilkes G. L.: Some investigations on the fiber formation by utilizing a side-by-side bicomponent electrospinning approach. Polymer, 44, 63536359 (2003).

DOI: $10.1016 / \mathrm{S} 0032-3861(03) 00616-5$

[20] Chen H-M., Yu D-G.: An elevated temperature electrospinning process for preparing acyclovir-loaded PAN ultrafine fibers. Journal of Materials Processing Technology, 210, 1551-1555 (2010).

DOI: 10.1016/j.jmatprotec.2010.05.001

[21] Wu Y., Yu J-Y., He J-H., Wan Y-Q.: Controlling stability of the electrospun fiber by magnetic field. Chaos Solitons Fractals, 32, 5-7 (2007).

DOI: $10.1016 /$ j.chaos.2006.05.023

[22] Liu Y., He J-H.: Bubble electrospinning for mass production of nanofibers. International Journal of Nonlinear Sciences and Numerical Simulation, 8, 393-396 (2007).
[23] Tripatanasuwan S., Zhong Z., Reneker D. H.: Effect of evaporation and solidification of the charged jet in electrospinning of poly(ethylene oxide) aqueous solution. Polymer, 48, 5742-5746 (2007).

DOI: $10.1016 /$ j.polymer.2007.07.045

[24] Hsu C-M., Shivkumar S.: Nano-sized beads and porous fiber constructs of poly( $\varepsilon$-caprolactone) produced by electrospinning. Journal of Materials Science, 39, 3003-3013 (2004).

DOI: 10.1023/B:JMSC.0000025826.36080.cf

[25] Kanjanapongkul K., Wongsasulak S., Yoovidhya T.: Investigation and prevention of clogging during electrospinning of zein solution. Journal of Applied Polymer Science, 118, 1821-1829 (2010).

DOI: $10.1002 /$ app.32499

[26] Li Y., Lim L-T., Kakuda Y.: Electrospun zein fibers as carriers to stabilize (-)-epigallocatechin gallate. Journal of Food Science, 74, 233-240 (2009). DOI: $10.1111 /$ j.1750-3841.2009.01093.x

[27] Yu D-G., Branford-White C., White K., Li X-L., Zhu L-M.: Dissolution improvement of electrospun nanofiber-based solid dispersions for acetaminophen. AAPS PharmSciTech, 22, 809-817 (2010). DOI: $10.1208 / \mathrm{s} 12249-010-9438-4$

[28] Yu D. G., Yang J. M., Branford-White C., Lu P., Zhang L., Zhu L. M.: Third generation solid dispersions of ferulic acid in electrospun composite nanofibers. International Journal of Pharmaceutics, 400, 158-164 (2010). DOI: $10.1016 /$ j.ijpharm.2010.08.010

[29] Yu D-G., Zhang X-F., Shen X-X., Branford-White C., Zhu L-M.: Ultrafine ibuprofen-loaded polyvinylpyrrolidone fiber mats using electrospinning. Polymer International, 58, 1010-1013 (2009).

DOI: $10.1002 /$ pi.2629

[30] Bühler V.: Polyvinylpyrrolidone excipients for pharmaceuticals: Povidone, crospovidone and copovidone. Springer, Berlin (2005).

[31] Crowley J. M.: Role of Joule heating in the electrostatic spraying of liquids. Journal of Applied Physics, 48, 145-147 (1977).

[32] Chew S. Y., Hufnagel T. C., Lim C. T., Leong K. W.: Mechanical properties of single electrospun drugencapsulated nanofibres. Nanotechnology, 17, 38803891 (2006).

DOI: $10.1088 / 0957-4484 / 17 / 15 / 045$

[33] Moon J., Park J-A., Lee S-J., Lim S. C., Zyung T.: Structure and electrical properties of electrospun $\mathrm{ZnO}-\mathrm{NiO}$ mixed oxide nanofibers. Current Applied Physics, 9, 213-216 (2009).

DOI: $10.1016 /$ j.cap.2008.12.020

[34] Reneker D. H., Yarin A. L.: Electrospinning jets and polymer nanofibers. Polymer, 49, 2387-2425 (2008). DOI: $10.1016 /$ j.polymer.2008.02.002 